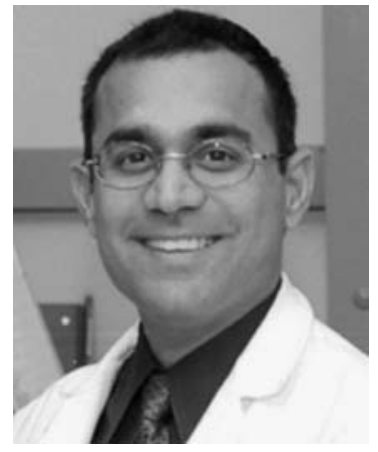

\title{
A Conversation with Raghu Kalluri
}

\author{
InTeRviewer: Gemma Alderton
}

Senior Editor, Nature Reviews Cancer

Raghu Kalluri is Chair of the Department of Cancer Biology and Director of the Metastasis Research Center at the University of Texas MD Anderson Cancer Center.

Gemma Alderton: Previously, you've worked on fibrosis and fibroblasts, but you've moved recently into this field of exosomes.

Dr. Kalluri: I've worked in the area of tumor microenvironment for many years now. Work on exosomes is a logical extension because if you look at the tumor microenvironment, in addition to cells and extracellular proteins, there are also these little vesicles that are part of the extracellular milieu. Therefore we asked what these vesicles do and whether there is anything valuable we can learn from them to diagnose or treat cancer.

Gemma Alderton: Where are we at with regard to the diagnostic work in the field?

Dr. Kalluri: Obviously, the whole area of liquid biopsy is very big now. The idea that we can harness information about cancer from the bloodstream or any body fluid is very attractive because you don't have to keep probing tumor tissue or metastatic tissue. There's a lot of information that is carried in the blood via exosomes and freecirculating material. We are excited about exosomes because they come from live cells. That means that you get a reflection of what the cell is undergoing: whether it is stressed or not stressed, whether it has mutations in it causing it to have pathways that are altered. All of that will reflect in the exosomes coming from that cell, and that information can be useful to understand the behavior of the cell, diagnose cancer, and understand how that information could be used for therapy. In the next 5 years you will see serum genomics, body fluid genomics, and liquid-biopsy-associated biomarkers to be of great value to not only diagnose disease but also to be able to rapidly track if a therapy has worked or not.

Gemma Alderton: Could you detect cancers earlier than with imaging?

Dr. Kalluri: Potentially. Let's say you were to take blood from somebody who is 60 years old. If you have done enough studies, you could know, if you isolate the exo- somes from this person's blood, which exosomes could predict if there is a certain type of cancer somewhere in the body. Even if you're not absolutely sure, it will give you some risk factor assessment. Then you can follow that up with imaging and other things. I think that's going to be the wave of the future. Of course, I think that early detection and prevention is probably the only way that we will beat cancer, not just treating an advanced disease.

Gemma Alderton: Cancer cells are not the only cells that produce exosomes, so a challenge in the field is determining which exosomes from a blood sample come from a cancer cell.

Dr. Kalluri: There is, of course, a need to distinguish anything coming from cancer cells from anything coming from other cells in the blood. Once we've done that though, when you identify those distinctions in a collection of everything, you know it's coming from cancer cells. That's one way we will overcome that. For example, you can take free-circulating DNA or even exosomes in the blood and do deep sequencing and you can find information. To make it really broadly available to everybody, you have to bring the cost down, and the technology should be very minimal to all centers that do this diagnosis. You're correct though, in that you have to find a way to enrich the sample for something coming from cancer cells, and we've done that. We found certain proteins that are present on exosomes coming from cancer cells but not from normal cells. That allows for an enrichment process that lets us look at that material inside the exosome and be more certain that it's coming from cancer cells. That allowed us to predict things earlier, at least in pancreatic cancer.

Gemma Alderton: Were these markers on the exosomes specific to pancreatic cancer? Do other types of cancer have different markers that would have to be identified separately?

Dr. Kalluri: My gut feeling, and not having any data to back it up right now, is that there will be some markers

(C) 2016 Kalluri. This article is distributed under the terms of the Creative Commons Attribution-NonCommercial License, which permits reuse and redistribution, except for commercial purposes, provided that the original author and source are credited. 
coming from all types of cancer cells - just because there are certain common pathways that trigger in most cancer cells that will create proteins in those pathways - that will probably be on or in the exosomes. There are going to be some specific ones, but I'm more interested in the common ones. The protein we found seems to be a common one, but it tends to recognize exosomes coming from pancreatic cancer cells much better. That doesn't mean it doesn't do well with other cancers. It's just that it's not as robust and perfect as with pancreatic cancer samples. We have to do these studies systematically with other cancers to see if there's anything unique we can find. Overall, I'm optimistic that something more generic will be found.

The interest in exosomes is not just to have it on the surface so you can use it as a biomarker but also to enrich it so you can appreciate the microRNA in it, the mRNA in it, the DNA in it, the metabolites that are reflective of cancer cells. You're increasing the signal compared with background.

Gemma Alderton: A lot of questions today are about exosome biogenesis and how the huge plethora of proteins and nucleic acids found in exosomes arises.

Dr. Kalluri: The biogenesis of exosomes is pretty much an open field. There have been some studies done, some proteins identified. There is some good science there, but the bulk of it is unknown. The reason for that is that there was some simplification of the nomenclature, with exosomes being a particular type of entity, and extracellular vesicles being another entity and microvesicles yet another, all because of their size differences. We have a standard way of making exosomes. Somebody wants to call them "extracellular vesicles"? That's fine. One day, we will have to understand if there are in fact different populations doing different things within this collection of exosomes. For now, not having access to such tools, we collect all of them and we study them together. How all of these come about in a cell to be secreted, nobody knows. There could be five different ways of doing it, or ten, or maybe only one way.

Gemma Alderton: Might that be an interesting avenue to explore in terms of preventing biogenesis, particularly from cancer cells?

Dr. Kalluri: I would think so. If you prevent cancer cells from making exosomes, what would be the impact on tumor progression and in metastasis? That requires us to do more experiments, to understand what specific proteins or collection of proteins are important to suppress exosome production without altering other pathways, then asking that question. There are some proteins that tend to have some effect on biogenesis, but again, if it's a heterogeneous population, then you're only going to inhibit one way of producing exosomes but not the other way.

Gemma Alderton: There's been lots of discussion about using exosomes to target genes or proteins in cells.
Dr. Kalluri: That's an exciting area, because there's been enough research done to suggest that if we can enclose drugs or particles and get them to deliver that drug to a particular place in the body, it's a great way to have specific therapies for specific tissues and not have side effects. We have trillions, maybe even a quadrillion, of exosomes floating in our blood all through our body, and they tend to travel. They go into tissues. They enter cells. Why not use this natural property to send drugs? That's what we're pursuing. We're trying to understand how we can put drugs in exosomes and use their natural ability to float around the blood and enter the cells that they would enter naturally. If they enter a cell where there's some specific inhibition that needs to happen through a drug, then they'll have an impact.

Right now, the goal is to see if we can deliver drugs using exosomes. Once that happens, then we can ask "How efficient it is? Is it more efficient than liposomes or nanoparticles carrying these drugs?" It turns out that they are, because exosomes tend to enter cells more efficiently. They have so many other surface proteins on them that enable them to fuse with the plasma membrane, enter the cell, and dump whatever drug cargo they have. They are selected for cancer cells because cancer cells tend to take things in more readily than normal cells because of certain oncogenic events that are amplified. That particular property of cancer cells allows exosomes to enter them more readily.

Gemma Alderton: How do you make the exosomes to package them? Do you take them from the patients to individualize them?

Dr. Kalluri: You could take them from patients, but you could even take them from cells like fibroblasts, which, if you inject them in mice, don't do much. There doesn't seem to be any toxicity associated with them. You can make them from any cell type that you think is benign enough to not cause any problems. You can personalize it for the patient, and then send it back. For cells, it's easy. You could make billions of exosomes in a day, so you could create the drug payload very easily.

One of the most exciting things is that we already have ways like siRNAs or shRNAs to suppress bad transcripts that we don't want. The problem has been how to get them into the cancer cells. I think exosomes can solve that problem. Potentially, you could even deliver things that cells don't normally take in; exosomes will enter the cell and dump it in there. Certain drugs don't enter the cells, but exosomes could disguise them, take them inside, and deliver them to have an impact on something that is inside the cell. You could target the intracellular milieu with exosomes. You don't have to only target the surface of the cancer cell, like most monoclonal antibodies do.

Gemma Alderton: What sort of intracellular processes might you target?

Dr. Kalluri: Transcription factors. Many of them are critical for cancer and they're up-regulated. They're small 
molecules and notoriously difficult to target. You could use exosome technology to target them.

Gemma Alderton: What are some of the future challenges confronting the field?

Dr. Kalluri: With any young field like this you really need to do some fundamental biology. You need to understand why exosomes have what they have. Is it consistent? Does a cell in the morning make exosomes with a payload that is similar to a cell in the evening that is making exosomes? What is the variation? We need to understand some of the basic biology so that when we do experiments, we know that there's internal consistency. Moreover, it looks like pretty much everything that's found in the cell is found in the exosome, but we need to catalog that. For the next couple of years we're probably auditing and cataloging everything so that we know what these vesicles have and then what we can add to them to make them do what we need to do to treat cancer patients.

On the other hand, maybe exosomes coming from cancer cells can be inhibited. Understanding the biology and biogenesis of exosomes is probably going to be very important. That's a challenge because these are very small vesicles. They are not cells; it's hard to manipulate them. We have to come up with new imaging techniques, new way to manipulate them so that they can be generated in a way that we can understand how the composition of exosomes reflects specific manipulations of their cell of origin. These things are technology-dependent, but I think exosomes are going to be an exciting area of biology. In the next 5 to ten years, we'll be amazed by the things that exosomes are doing in our body and how they can be a reflection of some type of primordial particles that have evolved to provide complex functions in human beings. 


\section{$\$_{\text {CSH\& }}^{\infty}$ Cold Spring Harbor Symposia SYMPOSIA}

\section{A Conversation with Raghu Kalluri}

Cold Spring Harb Symp Quant Biol 2016 81: 317-319 originally published online April 19, 2017 Access the most recent version at doi:10.1101/sqb.2016.81.033043

Creative This article is distributed under the terms of the

Commons http://creativecommons.org/licenses/by-nc/4.0/, which permits reuse and

License redistribution, except for commercial purposes, provided that the original author and source are credited.

Email Alerting Receive free email alerts when new articles cite this article - sign up in Service the box at the top right corner of the article or click here. 